\title{
Exploring the timelike region for the elastic form factor in the light-front quantization
}

\author{
Ho-Meoyng Choi and Chueng-Ryong Ji \\ Department of Physics, North Carolina State University, Raleigh, N.C. 27695-8202
}

\begin{abstract}
Even though the Drell-Yan-West formulation is the most rigorous and well-established framework to compute the exclusive processes, its utility has been limited only to the spacelike region because of the intrinsic kinematic constraint $q^{+}=0$. We present an explicit example demonstrating how one may obtain the necessary information (i.e. nonvalence or so called Z-graph contribution) in the timelike region of exclusive process without encountering a formidable task of direct calculation that has hindered so far the progress in this area. In the analysis of $q \bar{Q}$ bound state form factors using an exactly solvable model of $(3+1)$ dimensional scalar field theory interacting with gauge fields, the results analytically continued from the spacelike region coincide exactly with the direct results in the timelike region. This example verifies that the method of analytic continuation is capable of yielding the effect of complicate nonvalence contributions. The meson peaks analogous to the vector meson dominance(VMD) phenomena are also generated at the usual VMD positions.
\end{abstract}

PACS: 11.15.Bt, 11.10.Kk, 11.40.-q

Keywords: Time-like form factor, Analytic continuation, Dispersion relation 


\section{INTRODUCTION}

The Drell-Yan-West $\left(q^{+}=q^{0}+q^{3}=0\right)$ frame in the light-front quantization provided an effective formulation for the calculation of various form factors in the spacelike momentum transfer region $q^{2}=-Q^{2}<0$ [1]. As an example, only parton-number-conserving Fock state (valence) contribution (e.g. Fig. 1(a)) is needed in $q^{+}=0$ frame when the "good" components of the current, $j^{+}$and $j_{\perp}=\left(j_{x}, j_{y}\right)$, are used [2] for the spacelike electromagnetic form factor calculation of pseudoscalar mesons. Successful light-front quark model (LFQM) description of various pseudoscalar form factors can also be found in the literatures [3] 7 . However, not all is well. For the higher spin system composed of fermions, one needs in general to consider the nonvalence contribution (e.g. Fig. 1(b)) to fulfill the covariance requirement even when $j^{+}$and $j_{\perp}$ are used in the $q^{+}=0$ frame. The nonvalence contribution in the $q^{+}=0$ frame is called the "zero-mode". The details of zero-mode contributions for

the spin-one system with the $j^{+}$current coupled with longitudinal polarization vector as well as the semileptonic transition form factor of pseudoscalar meson with the $j_{\perp}$ current can be seen in Refs. [8 [10].

Furthermore, the timelike $\left(q^{2}>0\right)$ form factor analysis in the light-front quark model has been hindered by the fact that $q^{+}=0$ frame is defined only in the spacelike region $\left(q^{2}=q^{+} q^{-}-q_{\perp}^{2}<0\right)$. While the $q^{+} \neq 0$ frame can be used in principle to compute the timelike form factors, it is inevitable (if $q^{+} \neq 0$ ) to encounter the nonvalence diagram arising from the quark-antiquark pair creation (so called "Z-graph") as shown in Fig. 1(b). The main source of difficulty in calculating the nonvalence diagram (Fig. 1(b)) is the lack of information on the black blob which should contrast with the white blob representing the usual light-front valence wave function. In fact, it was reported [3] that the omission of nonvalence contribution leads to a large deviation from the full results. The timelike form factors associated with the hadron pair productions in $e^{+} e^{-}$annihilations also involve the nonvalence contributions. Therefore, it is not a simple task to explore the timelike region with the framework at hand and the investigation is still just at the beginning stage to the 
best of our knowledge.

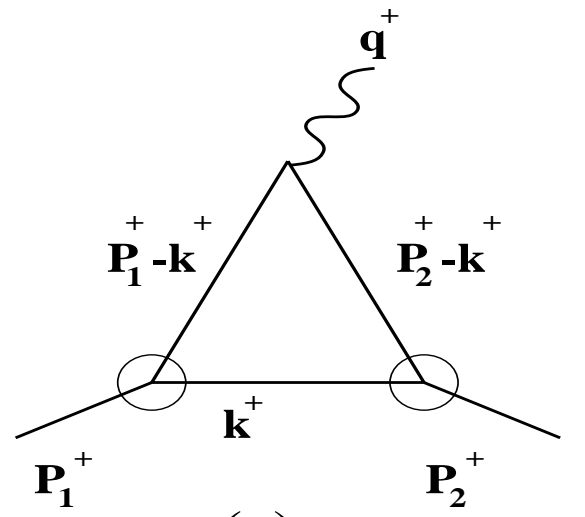

(a)

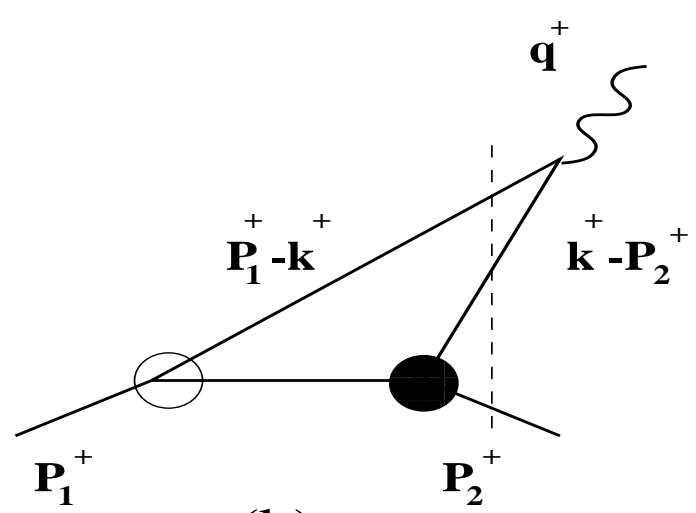

(b)

FIG. 1. The light-front quark model description of a electroweak meson form factor: (a) the usual light-front valence diagram and (b) the nonvalence(pair-creation) diagram. The vertical dashed line in (b) indicates the energy-denominator for the nonvalence contributions. While the white blob represents the usual light-front valence wave function, the modeling of black blob has not yet been made.

The aim of this paper is to provide at least a clear example of demonstration showing how one might take advantage of the existing formulation to get the new information (i.e. nonvalence contribution or so called Z-graph) necessary in the timelike region of exclusive processes. This can be done by the analytic continuation from the spacelike form factor calculated in the Drell-Yan-West $\left(q^{+}=0\right)$ frame to the timelike region. In this paper, we focus on the case that does not require the zero-mode contributions even though one can in principle apply the same method presented in this work to the case of zero-mode contributions once they are identified. However, in the case of the zero-mode contributions, an additional step is necessary to identify them凸.

\footnotetext{
${ }^{1}$ An example including the zero-mode contribution can be shown in the calculation of the $f_{-}$form factor for the exclusive semileptonic decays between two pseudoscalar mesons using the fermionic covariant model with the monopole smearing vertices [2,9]. In this case, we indeed verified the applicability of our method by checking if the identical results are obtained when different com-
} 
For an explicit demonstration of our work, we use an exactly solvable model of $(3+1)$ dimensional scalar field theory interacting with gauge fields. The Lagrangian of the system is given by [11]

$$
\begin{aligned}
\mathcal{L}= & \left(\partial_{\mu} \phi_{a}+i e_{a} A_{\mu} \phi_{a}\right)^{\dagger}\left(\partial^{\mu} \phi_{a}+i e_{a} A^{\mu} \phi_{a}\right)-m_{a}^{2} \phi_{a}^{\dagger} \phi_{a} \\
& +\left(\partial_{\mu} \phi_{b}+i e_{b} A_{\mu} \phi_{b}\right)^{\dagger}\left(\partial^{\mu} \phi_{b}+i e_{b} A^{\mu} \phi_{b}\right)-m_{b}^{2} \phi_{b}^{\dagger} \phi_{b} \\
& -\frac{1}{2}\left(M^{2} \Phi^{2}-\partial_{\mu} \Phi \partial^{\mu} \Phi\right)+g \Phi\left(\phi_{a}^{\dagger} \phi_{b}+\phi_{b}^{\dagger} \phi_{a}\right),
\end{aligned}
$$

where $\phi_{a(b)}$ corresponds to the bosonic quark field with the mass $m_{a(b)}$ and $\Phi$ is the bound state meson with the mass $M$. Our model is essentially the $(3+1)$ dimensional extension of Mankiewicz and Sawicki's $(1+1)$ dimensional quantum field theory model [12], which was later reinvestigated by several others [11, 13, [16]. The starting model wave function is the solution of covariant Bethe-Salpeter (BS) equation in the ladder approximation with a relativistic version of the contact interaction [12]. Here, we do not take the Hamiltonian approach. The covariant model wave function is a product of two free single particle propagators, the overall momentum-conservation Dirac delta, and a constant vertex function. Consequently, all our form factor calculations are nothing but various ways of evaluating the Feynman perturbation-theory triangle diagram in scalar field theory.

Even though the model may not be as realistic as one may want, it achieves the goal of being the first example up to our knowledge. We think that it provides at least some guidance how one can persue the same (or similar) idea in the more realistic (perhaps more phenomenological in that respect) models. In this model, we calculate: (A) the timelike process of $\gamma^{*} \rightarrow M+\bar{M}$ transition in $q^{+} \neq 0\left(q^{2}>0\right)$ frame, (B) the spacelike process of $M \rightarrow \gamma^{*}+M$ in $q^{+} \neq 0\left(q^{2}<0\right)$ frame, and (C) the spacelike process of $M \rightarrow \gamma^{*}+M$ in $q^{+}=0$ frame. Using the analytic continuation from $q^{2}<0$ to $q^{2}>0$, we show that the result in $(\mathrm{C})$, which is obtained without encountering the nonvalence contributions at all,

ponents of the current are used in different frames, i.e., $q^{+} \neq 0$ and $q^{+}=0$ frames. See also the discussion in Section V. 
exactly reproduces the result in (A). In fact, all three results $(\mathrm{A}),(\mathrm{B})$, and $(\mathrm{C})$ coincide with each other in the entire $q^{2}$ range. We also confirm that our results are consistent with the dispersion relations [17 20]. We consider not only for the equal quark/antiquark mass case such as the pion but also for the unequal mass cases such as $K$ and $D$.

The paper is organized as follows: In Sec. II, we derive the timelike electromagnetic (EM) form factor of $\gamma^{*} \rightarrow M+\bar{M}$ process in the $q^{+} \neq 0$ frame (A) and discuss the singularities occuring from the on-energy shell of quark-antiquark pair creation. In Sec. III, the spacelike form factor of $M \rightarrow \gamma^{*}+M$ process is calculated both in the $q^{+} \neq 0(\mathrm{~B})$ and $q^{+}=0$ (C) frames. We then analytically continue the spacelike form factors to the timelike region. The singularities occured in the timelike region are also discussed. In Sec. IV, for the numerical calculation of the $\operatorname{EM}(\pi, K$, and $D)$ meson form factors for three different cases $(\mathrm{A}),(\mathrm{B})$ and (C), we use the constituent quark and antiquark masses $\left(m_{u}=m_{d}=0.25 \mathrm{GeV}, m_{s}=0.48\right.$ $\mathrm{GeV}$, and $m_{c}=1.8 \mathrm{GeV}$ ) [7, 3,21] and show that the form factors obtained from those three different cases are indeed equal to each other for the entire $q^{2}$ region. The meson peaks analogous to the vector meson dominance(VMD) are also obtained. The conclusion and discussion follows in Sec. V.

\section{FORM FACTORS IN THE TIMELIKE REGION}

The EM local current $J^{\mu}(0)$ responsible for a virtual photon decay into two $q \bar{Q}$ bound states in the scalar field theory can be calculated using the diagrams shown in Fig. 2. The covariant diagram shown in Fig. 2(a) is equivalent to the sum of two light-front time-ordered diagrams in Figs. 2(b) and 2(c). The EM current $J^{\mu}(0)$ obtained from the covariant diagram of Fig. 2(a) is given by

$$
\begin{aligned}
J^{\mu}(0)= & i e_{q} g^{2} \int d^{4} k \frac{1}{(q-k)^{2}-m_{q}^{2}+i \epsilon}(q-2 k)^{\mu} \frac{1}{\left(q-k-P_{2}\right)^{2}-m_{\bar{Q}}^{2}+i \epsilon} \\
& \times \frac{1}{k^{2}-m_{q}^{2}+i \epsilon}+e_{\bar{Q}}\left(m_{q} \leftrightarrow m_{\bar{Q}} \text { of the first term }\right),
\end{aligned}
$$

where $m_{q(\bar{Q})}$ and $e_{q(\bar{Q})}$ are the constituent quark (antiquark) mass and charge, respectively. 


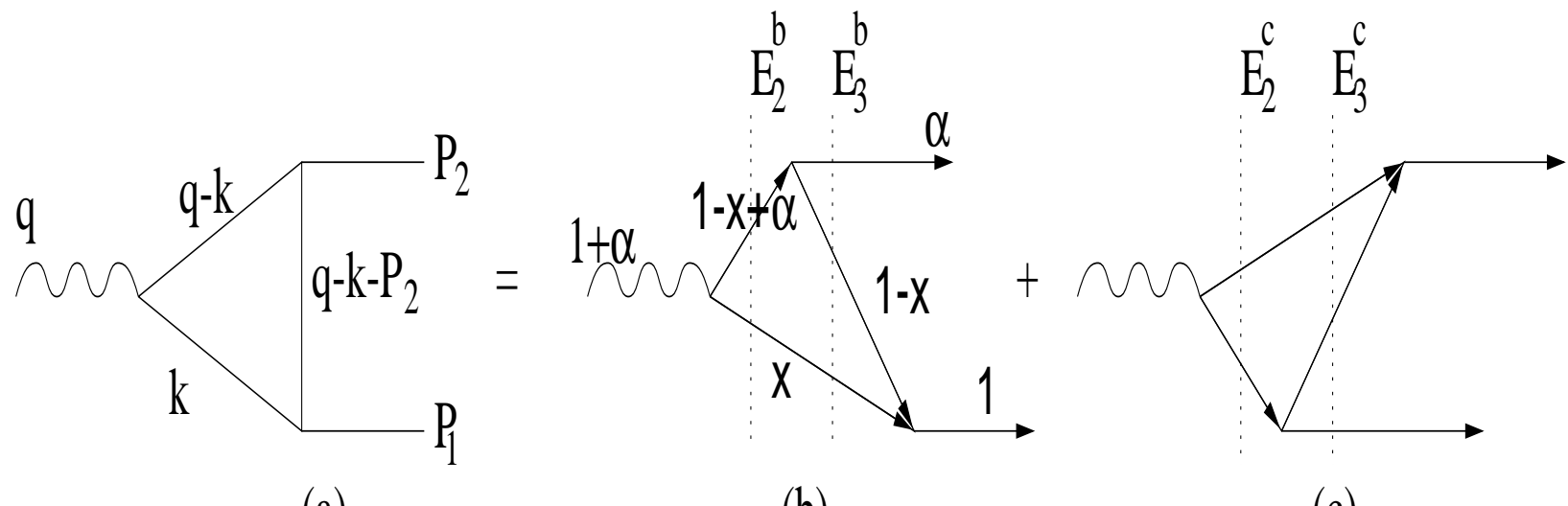

(a)

(b)

(c)

FIG. 2. The electromagnetic decays of a photon into two-body bound sates, i.e., $\gamma^{*} \rightarrow q \bar{q}$ (or $Q \bar{Q}) \rightarrow \mathcal{M}(q \bar{Q}) \mathcal{M}(q \bar{Q})$, in scalar theory: Covariant representation (a), and the light-front time ordered contributions to the decay amplitude (b) and (c).

The corresponding form factor $F\left(q^{2}\right)$ of the $q \bar{Q}$ bound state in timelike $\left(q^{2}>0\right)$ region is defined by

$$
J^{\mu}(0)=\left(P_{1}-P_{2}\right)^{\mu} F\left(q^{2}\right)
$$

where $q=P_{1}+P_{2}, P_{1}^{2}=P_{2}^{2}=M^{2}$ and $M$ is the mass of a $q \bar{Q}$ bound state scalar particle.

Using the Cauchy integration over $k^{-}$in Eq. (2), we can find each time-ordered contribution (Figs. 2(b) and 2(c)) to the timelike form factor $F\left(q^{2}>4 M^{2}\right)$ in Eq. (3). This procedure allows us to analyze the singularity structure of each light-front time-ordered diagram as well. For the calculation of each light-front time-ordered contribution, we take the purely longitudinal momentum frame, i.e., $q^{+} \neq 0, q_{\perp}=0$ and $P_{1 \perp}=P_{2 \perp}=0$. Accordingly, $q^{2}\left(=q^{+} q^{-}\right)>4 M^{2}$ is given by

$$
q^{2}=M^{2}(1+\alpha)^{2} / \alpha
$$

where $\alpha=P_{2}^{+} / P_{1}^{+}=q^{+} / P_{1}^{+}-1$ is the longitudinal momentum fraction and the two solutions for $\alpha$ are given by

$$
\alpha_{ \pm}=\left(\frac{q^{2}}{2 M^{2}}-1\right) \pm \sqrt{\left(\frac{q^{2}}{2 M^{2}}-1\right)^{2}-1}
$$

Note that both $\alpha_{ \pm}=1$ correspond to the threshold, $q^{2}=4 M^{2}$. The EM form factor $F\left(q^{2}\right)$ in Eq. (3) is independent of the subscript sign of $\alpha$. Thus, one can take either $\alpha_{+}$or $\alpha_{-}$ 
to calculate $F\left(q^{2}\right)$. Here, for convenience, we use $\alpha=\alpha_{-}$which ranges from 0 to 1 for the physical momentum transfer region, i.e., $\alpha_{-} \rightarrow 0$ as $q^{2} \rightarrow \infty$ and 1 as $q^{2} \rightarrow 4 M^{2}$. Of course, we can use $\alpha=\alpha_{+}$equally well and verify that the two results $\left(\alpha_{+}\right.$and $\left.\alpha_{-}\right)$are exactly same for the calculation of $F\left(q^{2}\right)$.

Since $q^{+}>P_{1}^{+} \geq P_{2}^{+}>0$ for $\alpha=\alpha_{-}$, the Cauchy integration over $k^{-}$in Eq. (2) has two nonzero contributions to the residue calculations, one coming from the interval (i) $0<k^{+}<P_{1}^{+}$(see Fig. 2(b)) and the other from (ii) $P_{1}^{+}<k^{+}<q^{+}$(see Fig. 2(c)). The internal momentum $k^{+}$is defined by $k^{+}=x P_{1}^{+}$, where $x$ is the Lorentz invariant longitudinal momentum variable. The "good" component of the current, $J^{+}(0)$, is used in our computation of the two light-front diagrams Figs. 2(b) and 2(c). In the following, for simplicity, we will explicitly write neither the obvious second term in Eq. (2) nor the charge factor $\left(e_{q}\right.$ or $\left.e_{\bar{Q}}\right)$.

In the region of $0<k^{+}<P_{1}^{+}$, the residue is at the pole of $k^{-}=\left[m_{q}^{2}+k_{\perp}^{2}-i \epsilon\right] / k^{+}$, which is placed in the lower half of complex- $k^{-}$plane. Thus, the Cauchy integration of $J^{+}$ in Eq. (2) over $k^{-}$in this region gives

$$
\begin{aligned}
J_{b}^{+}(0)= & \pi g^{2} \int_{0}^{P_{1}^{+}} d k^{+} d^{2} k_{\perp} \frac{q^{+}-2 k^{+}}{k^{+}\left(q^{+}-k^{+}\right)\left(P_{1}^{+}-k^{+}\right)} \\
& \times \frac{1}{\left[q^{-}-\left(m_{q}^{2}+k_{\perp}^{2}\right) / k^{+}-\left(m_{q}^{2}+k_{\perp}^{2}\right) /\left(q^{+}-k^{+}\right)\right]} \\
& \times \frac{1}{\left[q^{-}-P_{2}^{-}-\left(m_{q}^{2}+k_{\perp}^{2}\right) / k^{+}-\left(m_{\bar{Q}}^{2}+k_{\perp}^{2}\right) /\left(P_{1}^{+}-k^{+}\right)\right]},
\end{aligned}
$$

where the subscript $b$ in $J_{b}^{+}(0)$ implies the current in Fig. 2(b) and $g$ is fixed by the normalization of the EM form factor at $q^{2}=0$. The last two terms in Eq. (6) correspond to the two and three particle intermediate states. We represent the energy denominators of the two and three particle intermediate states as $E_{2}^{b}$ and $E_{3}^{b}$ in Fig. 2(b), respectively.

To analyze the singularities of Eq. (6), we further integrate over $k_{\perp}$ and obtain

$$
J_{b}^{+}(0)=\pi^{2} g^{2} \int_{0}^{1} d x \frac{x(1+\alpha-2 x) /(1+\alpha)}{\mathcal{E}_{3}^{b}-\mathcal{E}_{2}^{b}} \ln \left(\frac{\mathcal{E}_{2}^{b}}{\mathcal{E}_{3}^{b}}\right),
$$

where $\mathcal{E}_{2}^{b}=x(1+\alpha-x) M^{2} / \alpha-m_{q}^{2}$ and $\mathcal{E}_{3}^{b}=x(1-x) M^{2}-\left[x m_{q}^{2}+(1-x) m_{\bar{Q}}^{2}\right]$. While $\mathcal{E}_{3}^{b}$ is 
not zero $\left(\mathcal{E}_{3}^{b} \neq 0\right)$ in general for the entire physical region, $\mathcal{E}_{2}^{b}$ can be zero when $q^{2} \geq 4 m_{q(\bar{Q})}^{2}$. The singular structure of $\mathcal{E}_{3}^{b}-\mathcal{E}_{2}^{b}$ term in Eq. (可) depends on whether a $q \bar{Q}$ bound state scalar particle is strongly bounded $\left(M^{2}<m_{q}^{2}+m_{\bar{Q}}^{2}\right)$ or weakly bounded $\left(M^{2}>m_{q}^{2}+m_{\bar{Q}}^{2}\right)$. As we will show in our numerical calculations (Section IV), anomalous threshold appears for $M^{2}>m_{q}^{2}+m_{\bar{Q}}^{2}$ while only the normal threshold of bound state exists for $M^{2}<m_{q}^{2}+m_{\bar{Q}}^{2}$.

In the region of $P_{1}^{+}<k^{+}<q^{+}$, the residue is at the pole of $k^{-}=q^{-}-\left[m_{q}^{2}+\left(q_{\perp}-k_{\perp}\right)^{2}-\right.$ $i \epsilon] /\left(q^{+}-k^{+}\right)$, which is placed in the upper half of complex- $k^{-}$plane. Thus, the Cauchy integration of $J^{+}(0)$ in Eq. (2) over $k^{-}$in this region yields the result

$$
\begin{aligned}
J_{c}^{+}(0)= & -\pi g^{2} \int_{P_{1}^{+}}^{q^{+}} d k^{+} d^{2} k_{\perp} \frac{q^{+}-2 k^{+}}{k^{+}\left(q^{+}-k^{+}\right)\left(P_{1}^{+}-k^{+}\right)} \\
& \times \frac{1}{\left[q^{-}-\left(m_{q}^{2}+k_{\perp}^{2}\right) / k^{+}-\left(m_{q}^{2}+k_{\perp}^{2}\right) /\left(q^{+}-k^{+}\right)\right]} \\
& \times \frac{1}{\left[q^{-}-P_{1}^{-}+\left(m_{\bar{Q}}^{2}+k_{\perp}^{2}\right) /\left(P_{1}^{+}-k^{+}\right)-\left(m_{q}^{2}+k_{\perp}^{2}\right) /\left(q^{+}-k^{+}\right)\right]},
\end{aligned}
$$

where the subscript $c$ in $J_{c}^{+}(0)$ means the current in Fig. 2(c). After the integration over the $k_{\perp}$ in Eq. (8), we obtain

$$
J_{c}^{+}(0)=-\pi^{2} g^{2} \int_{0}^{1} d X \frac{\alpha X(1+\alpha-2 \alpha X) /(1+\alpha)}{\mathcal{E}_{3}^{c}-\mathcal{E}_{2}^{c}} \ln \left(\frac{\mathcal{E}_{2}^{c}}{\mathcal{E}_{3}^{c}}\right),
$$

where $x=1+\alpha(1-X), \mathcal{E}_{3}^{c}=X(1-X) M^{2}-\left[X m_{\bar{Q}}^{2}+(1-X) m_{q}^{2}\right]$ and $\mathcal{E}_{2}^{c}=\alpha X(1+\alpha-$ $\alpha X) M^{2} / \alpha-m_{q}^{2}$. The pole structure in Eq. (9) is equivalent to that of Eq. (77).

Consequently, the timelike form factor in Eq. (3) is given by

$$
\begin{aligned}
F\left(q^{2}\right)= & \frac{\pi^{2} g^{2}}{\alpha^{2}-1} \int_{0}^{1} d x\left\{\frac{x(1+\alpha-2 x)}{\mathcal{E}_{3}^{b}-\mathcal{E}_{2}^{b}} \ln \left(\frac{\mathcal{E}_{2}^{b}}{\mathcal{E}_{3}^{b}}\right)\right. \\
& \left.-\frac{\alpha x(1+\alpha-2 \alpha x)}{\mathcal{E}_{3}^{c}-\mathcal{E}_{2}^{c}} \ln \left(\frac{\mathcal{E}_{2}^{c}}{\mathcal{E}_{3}^{c}}\right)\right\},
\end{aligned}
$$

where $\mathcal{E}_{2}^{b}, \mathcal{E}_{3}^{b}, \mathcal{E}_{2}^{c}$ and $\mathcal{E}_{3}^{c}$ are defined in Eqs. (7) and (9). 


\section{FORM FACTORS IN SPACELIKE REGION AND THE ANALYTIC CONTINUATION TO THE TIMELIKE REGION}

In this section, we calculate the EM form factor in spacelike momentum transfer region and then analytically continue to the timelike region to compare the result with the timelike form factor (i.e. Eq. (10)) that we obtained in the previous section. The EM current of a $q \bar{Q}$ bound state in spacelike momentum transfer region is defined by the local current $j^{\mu}(0)$;

$$
j^{\mu}(0)=\left(P_{1}+P_{2}\right)^{\mu} \mathcal{F}\left(q^{2}\right),
$$

where $q=P_{1}-P_{2}, q^{2}<0$ and $\mathcal{F}\left(q^{2}\right)$ is the spacelike form factor. The EM current $j^{\mu}(0)$ obtained from the covariant triangle diagram of Fig. 3(a) is given by

$j^{\mu}(0)=i g^{2} \int d^{4} k \frac{1}{\left(P_{1}-k\right)^{2}-m_{q}^{2}+i \epsilon}\left(P_{1}+P_{2}-2 k\right)^{\mu} \frac{1}{\left(P_{2}-k\right)^{2}-m_{q}^{2}+i \epsilon} \frac{1}{k^{2}-m_{\bar{Q}}^{2}+i \epsilon}$.

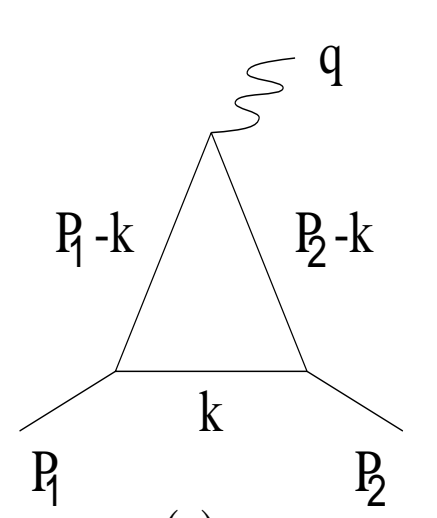

(a)

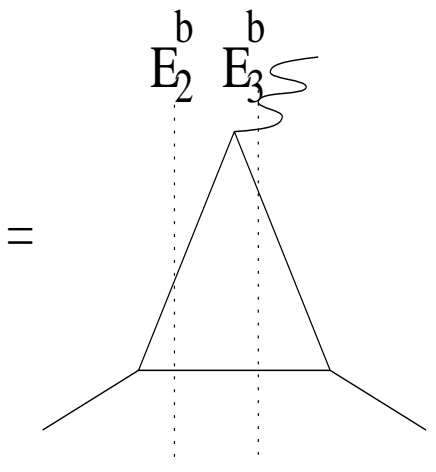

(b)

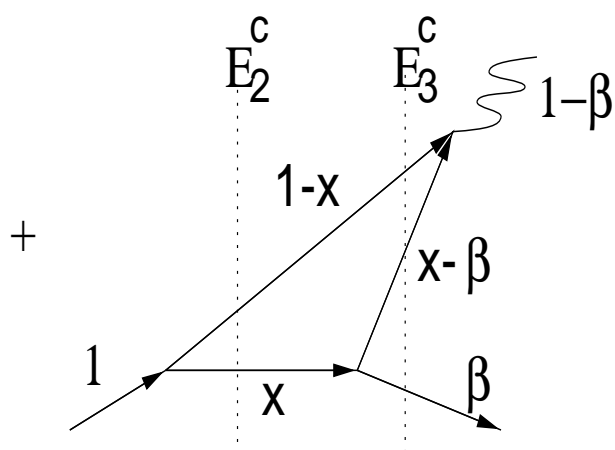

(c)

FIG. 3. Covariant triangle diagram (a) is represented as the sum of light-front triangle diagram (b) and the light-front pair-creation diagram (c).

As in the case of the timelike form factor in Sec. II, the Cauchy integration of $k^{-}$in Eq. (12) has also two contributions to the residue calculations, one coming from the interval $0<k^{+}<P_{2}^{+}$(see Fig. 3(b)) and the other from $P_{2}^{+}<k^{+}<P_{1}^{+}$(see Fig. 3(c)). Again, only the "good"-current $j^{+}(0)$ in Eq. (12) is used to obtain the contributions from Figs. 3(b) and $3(\mathrm{c})$. 
In the region of $0<k^{+}<P_{2}^{+}$, the residue is at the pole of $k^{-}=\left[m_{\bar{Q}}^{2}+k_{\perp}^{2}-i \epsilon\right] / k^{+}$, which is placed in the lower half of complex- $k^{-}$plane. Thus, the Cauchy integration of $j^{+}$ in Eq. (12) over $k^{-}$in this region yields

$$
\begin{aligned}
j_{b}^{+}(0)= & \pi g^{2} \int_{0}^{P_{2}^{+}} d k^{+} d^{2} k_{\perp} \frac{\left(P_{1}+P_{2}-2 k\right)^{+}}{\left(P_{1}-k\right)^{+}\left(P_{1}-k-q\right)^{+} k^{+}} \\
& \times \frac{1}{\left[P_{1}^{-}-\left[m_{q}^{2}+k_{\perp}^{2}\right] /\left(P_{1}^{+}-k^{+}\right)-\left[m_{\bar{Q}}^{2}+k_{\perp}^{2}\right] / k^{+}\right]} \\
& \times \frac{1}{\left[P_{1}^{-}-q^{-}-\left[m_{q}^{2}+\left(k_{\perp}+q_{\perp}\right)^{2}\right] /\left(P_{1}^{+}-k^{+}\right)-\left[m_{\bar{Q}}^{2}+k_{\perp}^{2}\right] / k^{+}\right]},
\end{aligned}
$$

where the subscript $b$ in $j_{b}^{+}(0)$ implies the current in Fig. 3(b).

In the region of $P_{2}^{+}<k^{+}<P_{1}^{+}$, the residue is at the pole of $k^{-}=P_{1}^{-}-\left[m_{q}^{2}+k_{\perp}^{2}-\right.$ $i \epsilon] /\left(P_{1}^{+}-k^{+}\right)$, which is placed in the upper half of complex- $k^{-}$plane. Thus, the Cauchy integration of $j^{+}$in Eq. (12) over $k^{-}$in this region becomes

$$
\begin{aligned}
j_{c}^{+}(0)= & \pi g^{2} \int_{P_{2}^{+}}^{P_{1}^{+}} d k^{+} d^{2} k_{\perp} \frac{\left(P_{1}+P_{2}-2 k\right)^{+}}{\left(P_{1}-k\right)^{+}\left(P_{1}-k-q\right)^{+} k^{+}} \\
& \times \frac{1}{\left[P_{1}^{-}-\left[m_{q}^{2}+k_{\perp}^{2}\right] /\left(P_{1}^{+}-k^{+}\right)-\left[m_{\bar{Q}}^{2}+k_{\perp}^{2}\right] / k^{+}\right]} \\
& \times \frac{1}{\left[P_{2}^{-}-P_{1}^{-}+\left[m_{q}^{2}+k_{\perp}^{2}\right] /\left(P_{1}^{+}-k^{+}\right)-\left[m_{q}^{2}+\left(q_{\perp}+k_{\perp}\right)^{2}\right] /\left(P_{2}^{+}-k^{+}\right)\right]} .
\end{aligned}
$$

As one can see from Eq. (14), the nonvalence contribution (Fig. 3(c)) vanishes only in the $q^{+}=0$ frame. Note that, for the spin-one system composed of fermions, however, Equation (14) does not vanish even in $q^{+}=0$ frame when the longitudinal polarization vector is involved as mentioned in the introduction. In the following, we investigate the spacelike form factor $\mathcal{F}\left(q^{2}\right)$ given in Eq. (11) using both $q^{+} \neq 0$ and $q^{+}=0$ frames. We then analytically continue to the timelike region in order to compare the result with the direct calculation of the timelike form factor $F\left(q^{2}\right)$ presented in the previous section. 


\section{A. The purely longitudinal $\left(q^{+} \neq 0\right.$ and $\left.q_{\perp}=0\right)$ frame}

In the purely longitudinal momentum frame $q^{+} \neq 0, q_{\perp}=0$, and $P_{1 \perp}=P_{2 \perp}=0$, the momentum transfer $q^{2}=q^{+} q^{-}$can be written in terms of the longitudinal momentum fraction $\beta=P_{2}^{+} / P_{1}^{+}=1-q^{+} / P_{1}^{+}$;

$$
q^{2}=-M^{2}(1-\beta)^{2} / \beta \leq 0,
$$

where the two solutions of $\beta$ are given by

$$
\beta_{ \pm}=\left(1-\frac{q^{2}}{2 M^{2}}\right) \pm \sqrt{\left(1-\frac{q^{2}}{2 M^{2}}\right)^{2}-1} .
$$

The form factor $\mathcal{F}\left(q^{2}\right)$ in Eq. (11) is also independent of the subscript sign of $\beta$. However, the condition $P_{2}^{+} \leq P_{1}^{+}$was used in obtaining Eqs. (13) and (14) and thus here we use $\beta=\beta_{-}$ $(0 \leq \beta \leq 1)$ in spacelike region. As shown in Eqs. (12)-(14), the sum of valence (Fig. 3(b)) and nonvalence (Fig. 3(c)) diagrams is equivalent to the covariant triangle diagram in Fig. $3(\mathrm{a})$.

For the analysis of singularity structures, we integrate over $k_{\perp}$ and obtain from the valence contribution(Fig. 3(b));

$$
j_{b}^{+}(0)=\pi^{2} g^{2} \int_{0}^{1} d x \frac{\beta x(1+\beta-2 \beta x)}{\tilde{\mathcal{E}}_{3}^{b}-\tilde{\mathcal{E}}_{2}^{b}} \ln \left(\frac{\tilde{\mathcal{E}}_{2}^{b}}{\tilde{\mathcal{E}}_{3}^{b}}\right),
$$

where $\tilde{\mathcal{E}}_{3}^{b}=x(1-x) M^{2}-\left[x m_{q}^{2}+(1-x) m_{\bar{Q}}^{2}\right]$ and $\tilde{\mathcal{E}}_{2}^{b}=\beta x(1-\beta x) M^{2}-\left[\beta x m_{q}^{2}+(1-\beta x) m_{\bar{Q}}^{2}\right]$. It turns out that Eq. (17) has no singularities because $\tilde{\mathcal{E}}_{3}^{b} \neq 0, \tilde{\mathcal{E}}_{2}^{b} \neq 0$, and $\tilde{\mathcal{E}}_{3}^{b}-\tilde{\mathcal{E}}_{2}^{b} \neq 0$ for the entire $q^{2}$ region. On the other hand, the $k_{\perp}$ integration for the current $j_{c}^{+}(0)$ in Eq. (14) yields

$$
j_{c}^{+}(0)=\pi^{2} g^{2} \int_{0}^{1} d \mathcal{X} \frac{(1-\beta)^{2} \mathcal{X}(2 \mathcal{X}-1)}{\tilde{\mathcal{E}}_{2}^{c}-\tilde{\mathcal{E}}_{3}^{c}} \ln \left(\frac{\tilde{\mathcal{E}}_{3}^{c}}{\tilde{\mathcal{E}}_{2}^{c}}\right),
$$

where $x=1-(1-\beta) \mathcal{X}, \tilde{\mathcal{E}}_{3}^{c}=(1-\beta) \mathcal{X}[1-(1-\beta) \mathcal{X}] M^{2}-\left[(1-\beta) \mathcal{X} m_{\bar{Q}}^{2}+(1-(1-\beta) \mathcal{X}) m_{q}^{2}\right]$ and $\tilde{\mathcal{E}}_{2}^{c}=-(1-\beta)^{2} \mathcal{X}(1-\mathcal{X}) M^{2} / \beta-m_{q}^{2}$. While $\tilde{\mathcal{E}}_{2}^{c}$ corresponding to the energy denominator of the two particle intermediate state does not vanish both in spacelike and timelike region, $\tilde{\mathcal{E}}_{3}^{c}$ of the three-particle intermediate state is not zero only for the spacelike momentum transfer 
region. For the timelike region, $\tilde{\mathcal{E}}_{3}^{c}$ can be zero so that the singularities start at $q_{\min }^{2}=4 m_{q(\bar{Q})}^{2}$ for $\gamma^{*} q \bar{q}\left(\gamma^{*} Q \bar{Q}\right)$ vertex. The singularity structure of $\tilde{\mathcal{E}}_{2}^{c}-\tilde{\mathcal{E}}_{3}^{c}$ in Eq. (18) is the same as in the case of timelike form factor (Section II), following the condition of a $q \bar{Q}$ bound state.

The EM form factor $\mathcal{F}\left(q^{2}\right)$ in Eq. (11) of a $q \bar{Q}$ bound state in $q^{+} \neq 0$ frame is then obtained by

$$
\begin{aligned}
\mathcal{F}\left(q^{2}, q^{+} \neq 0\right)= & \frac{\pi^{2} g^{2}}{1+\beta} \int_{0}^{1} d x\left\{\frac{\beta x(1+\beta-2 \beta x)}{\tilde{\mathcal{E}}_{3}^{b}-\tilde{\mathcal{E}}_{2}^{b}} \ln \left(\frac{\tilde{\mathcal{E}}_{2}^{b}}{\tilde{\mathcal{E}}_{3}^{b}}\right)\right. \\
& \left.+\frac{(1-\beta)^{2} x(2 x-1)}{\tilde{\mathcal{E}}_{2}^{c}-\tilde{\mathcal{E}}_{3}^{c}} \ln \left(\frac{\tilde{\mathcal{E}}_{3}^{c}}{\tilde{\mathcal{E}}_{2}^{c}}\right)\right\},
\end{aligned}
$$

where $\tilde{\mathcal{E}}_{2}^{b}, \tilde{\mathcal{E}}_{3}^{b}, \tilde{\mathcal{E}}_{2}^{c}$ and $\tilde{\mathcal{E}}_{2}^{c}$ are defined in Eqs. (17) and (18). Here, $\beta$ is a function of $q^{2}$. According to the analytic continuation, the sign of $q^{2}$ in Eq. (19) must be changed from to + for the timelike region.

\section{B. The Drell-Yan-West $\left(q^{+}=0\right)$ frame}

In Drell-Yan-West frame, $q^{+}=0, q^{2}=-q_{\perp}^{2}$, and $P_{1 \perp}=P_{2 \perp}=0$, the ' + ' component of the current has only the valence contribution, i.e., $j_{b}^{+}(0)$ in Eq. (13). The current $j_{b}^{+}(0)$ in $q^{+}=0$ frame is given by

$$
j_{b}^{+}(0)=\pi g^{2} \int_{0}^{1} d x d^{2} \ell_{\perp} \frac{2 x(1-x)}{\left(\mathcal{A}-\ell_{\perp}^{2}-\xi^{2}\right)^{2}-4 \xi^{2} \ell_{\perp}^{2} \cos ^{2} \phi},
$$

where $\ell_{\perp}=k_{\perp}+x q_{\perp} / 2, \mathcal{A}=x(1-x) M^{2}-\left[x m_{q}^{2}+(1-x) m_{\bar{Q}}^{2}\right]$, and $\xi^{2}=x^{2} q_{\perp}^{2} / 4$. The angle $\phi(0 \leq \phi \leq 2 \pi)$ is defined by $\ell_{\perp} \cdot \mathbf{q}_{\perp}=\left|\ell_{\perp}\right|\left|\mathbf{q}_{\perp}\right| \cos \phi$.

Integrating Eq. (20) over $\phi$ and $\ell_{\perp}$, we obtain

$$
\begin{aligned}
j_{b}^{+}(0)= & -\frac{8 \pi^{2} g^{2}}{\sqrt{q_{\perp}^{2}\left(4 M^{2}+q_{\perp}^{2}\right)}} \int_{0}^{1} d x \frac{(1-x)}{\sqrt{\left(x-x_{+}\right)\left(x-x_{-}\right)}} \\
& \times \tanh ^{-1}\left[\sqrt{\frac{q_{\perp}^{2}}{4 M^{2}+q_{\perp}^{2}}} \frac{x}{\sqrt{\left(x-x_{+}\right)\left(x-x_{-}\right)}}\right]
\end{aligned}
$$

where

$$
x_{ \pm}=\frac{2\left(M^{2}-m_{q}^{2}+m_{\bar{Q}}^{2}\right)}{4 M^{2}+q_{\perp}^{2}} \pm \sqrt{\frac{4\left(M^{2}-m_{q}^{2}+m_{\bar{Q}}^{2}\right)^{2}}{\left(4 M^{2}+q_{\perp}^{2}\right)^{2}}-\frac{4 m_{\bar{Q}}^{2}}{\left(4 M^{2}+q_{\perp}^{2}\right)}} .
$$


The analytic continuation from spacelike to timelike region in the $q^{+}=0$ frame requires the change of $q_{\perp}$ to $i q_{\perp}$ in Eqs. (21) and (22). We note from Eqs. (21) and (22) that the result of the timelike region exhibits the same singularity structure as the direct analyses in $q^{+} \neq 0$ frame, i.e., Eqs. (7) and (9), even though the nonvalence contribution in Fig. 3(c) is absent here.

After some manipulation, we obtain the EM form factor of a $q \bar{Q}$ bound state in the $q^{+}=0$ frame as follows

$$
\begin{aligned}
\mathcal{F}\left(q^{2}, q^{+}=0\right)= & -\frac{4 \pi^{2} g^{2}}{\sqrt{q^{2}\left(q^{2}-4 M^{2}\right)}} \int_{\arcsin \left(-\frac{a}{b}\right)}^{\arcsin \left(\frac{1-a}{b}\right)} d \theta(1-a-b \sin \theta) \\
& \times \tanh ^{-1}\left[\sqrt{\frac{q^{2}}{q^{2}-4 M^{2}}} \frac{a+b \sin \theta}{i b \cos \theta}\right],
\end{aligned}
$$

where $a=\left(x_{+}+x_{-}\right) / 2, b=\left(x_{+}-x_{-}\right) / 2$, and $q^{2}=-q_{\perp}^{2}$. While the representations in Eqs. (19) and (23) look apparently different, the two formulas, Eqs. (19) and (23), turn out to be actually identical. As we will show explicitly in the next section of numerical calculations, all three results of Eqs. (10), (19) and (23) indeed coincide exactly in the entire $q^{2}$ range.

\section{NUMERICAL RESULTS}

For our numerical analysis of $\pi, K$, and $D$ meson form factors, we use the physical meson masses together with the following constituent quark and antiquark masses: $m_{u}=m_{d}=0.25$ $\mathrm{GeV}, m_{s}=0.48 \mathrm{GeV}$, and $m_{c}=1.8 \mathrm{GeV}$ [3, 27,21]. Since our numerical results of the EM form factors obtained from Eqs. (10), (19) and (23) turn out to be exactly same with each other for the entire $q^{2}$ region, only a single line is depicted in Figs. 4, 5 and 6 for the form factor calculations of $\pi, K$, and $D$ mesons, respectively.

In should be noted from our constituent masses that $M^{2}<m_{q}^{2}+m_{\bar{Q}}^{2}$ for $\pi$ and $K$ and $M^{2}>m_{q}^{2}+m_{\bar{Q}}^{2}$ for $D$ meson cases. As discussed in Ref. 18 for the analysis of the one-

particle matrix element of a scalar current, the sigularity for $M^{2}>m_{q}^{2}+m_{\bar{Q}}^{2}$ case starts at 


$$
q_{\min }^{2}=\frac{1}{m_{\bar{Q}(q)}^{2}}\left[m_{q(\bar{Q})}^{2}-\left(M-m_{\bar{Q}(q)}\right)^{2}\right]\left[\left(M+m_{\bar{Q}(q)}\right)^{2}-m_{q(\bar{Q})}^{2}\right]
$$

for $\gamma^{*} q \bar{q}\left(\gamma^{*} Q \bar{Q}\right)$ vertex, while the singularity for $M^{2}<m_{q}^{2}+m_{\bar{Q}}^{2}$ case starts on the positive $q^{2}$-axis at the threshold point $q_{\min }^{2}=4 m_{q(\bar{Q})}^{2}$ for $\gamma^{*} q \bar{q}\left(\gamma^{*} Q \bar{Q}\right)$ vertex. Our numerical results exhibit all of these threshold behaviors coming from the normal $(\pi, K)$ and anomalous $(D)$ cases. As a consistency check, we also compare our numerical results of the form factor $F\left(q^{2}\right)=\operatorname{Re} F\left(q^{2}\right)+i \operatorname{Im} F\left(q^{2}\right)$ with the dispersion relations given by

$$
\begin{aligned}
& \operatorname{Re} F\left(q^{2}\right)=\frac{1}{\pi} P \int_{-\infty}^{\infty} \frac{\operatorname{Im} F\left(q^{\prime 2}\right)}{q^{\prime 2}-q^{2}} d q^{\prime 2} \\
& \operatorname{Im} F\left(q^{2}\right)=-\frac{1}{\pi} P \int_{-\infty}^{\infty} \frac{\operatorname{Re} F\left(q^{2}\right)}{q^{\prime 2}-q^{2}} d q^{\prime 2}
\end{aligned}
$$

where $P$ indicates the Cauchy principal value.

In Fig. 4(a), we show the EM form factor of the pion for $-2 \mathrm{GeV}^{2} \leq q^{2} \leq 3 \mathrm{GeV}^{2}$. The imaginary part (the dotted line) of the form factor starts at $q_{\min }^{2}=4 m_{u(d)}^{2}=0.25 \mathrm{GeV}$, which is consistent with the condition for $M^{2}<m_{q}^{2}+m_{\bar{Q}}^{2}$ case. It is interesting to note that the square of the total form factor $\left|F_{\pi}\left(q^{2}\right)\right|^{2}$ (thick solid line) produces a $\rho$ meson-type peak near $q^{2} \sim M_{\rho}^{2}$. However, we do not claim that this model indeed reproduces all the features of the vector meson dominance (VMD) phenomena because the more realistic phenomenological models may have to incorporate the more complex mechanism including the final state interaction. More investigation along this line is under consideration. Nevertheless, this simple model is capable of generating the peak and the position of peak is quite consistent with the VMD.

In Fig. 4(b), we show the timelike form factor of the pion for the entire $q^{2}>0$ region and compare the imaginary part of our direct calculations (dotted line) obtained from Eqs. (10), (19), and (23) with the result (data of black dots) obtained from the dispersion relations given by Eq. (26). Our direct calculation is in an excellent agreement with the solution of the dispersion relations. Our results for the real part are also confirmed to be in complete agreement with the dispersion relations. For high $q^{2}$ region, the imaginary part of the form factor is dominant over the real part (thin solid line). 
In Fig. 5(a), we show the kaon form factor for $-2 \mathrm{GeV}^{2} \leq q^{2} \leq 5 \mathrm{GeV}^{2}$. The kaon also has the normal singularity. However, it has two thresholds for the imaginary parts; one is $q_{\min }^{2}=4 m_{u}^{2}$ and the other is $q_{\min }^{2}=4 m_{s}^{2}$. These lead to the humped shape (dotted line) of the imaginary part shown in Fig. 5(a). While we have in principle two vector-meson-type peaks (i.e. $\rho$ and $\phi$ ), one can see in Fig. 5(a) only $\phi$ meson-type peak for the timelike kaon EM form factor above the physical threshold at $q_{\text {min }}^{2}=4 M_{K}^{2}$. We also show in Fig. $5(\mathrm{~b})$ the imaginary part from our direct calculation is in an excellent agreement with the result (data of black dots) from the dispersion relations for the entire timelike $q^{2}$ region. Again, the imaginary part is predominant for high $q^{2}$ region.

In Fig. 6, we show the $D$ meson form factor for $-10 \mathrm{GeV}^{2} \leq q^{2} \leq 30 \mathrm{GeV}^{2}$. Unlike the normal threshold of $\pi$ and $K$ form factor calculations, the $D$ meson form factor shows anomalous thresholds according to Eq. (24), i.e., $q_{\text {min }}^{2} \sim 0.24 \mathrm{GeV}^{2}$ (compared to $4 m_{d}^{2}=0.25$ $\mathrm{GeV}^{2}$ for normal case) and $q_{\text {min }}^{2} \sim 12.4 \mathrm{GeV}^{2}$ (compared to $4 m_{c}^{2}=12.96 \mathrm{GeV}^{2}$ for normal case) for the $\gamma^{*}-\bar{d}$ and $\gamma^{*}-c$ vertices, respectively. Similar to the kaon case in Fig. 5, we also have two unphysical peaks, i.e., $\rho$ and $J / \psi(1 S)$ meson type peaks due to $\bar{d}$ and $c$ quarks, respectively. However, the timelike form factor of $D$ meson has no pole structure for the the physical $q^{2} \geq 4 M_{D}^{2}$ region. In all of these figures (Figs. 4-6), the numerical result of Eq. (23) obtained from $q^{+}=0$ frame without encountering the nonvalence diagram coincides exactly with the numerical results of Eqs. (10) and (19) obtained from $q^{+} \neq 0$ frame.

\section{CONCLUSION AND DISCUSSION}

While it is not a simple task to explore the timelike region with the framework at hand, we attempted in this work to provide a clear example demonstrating that one can compute the timelike form factor without encountering the nonvalence contributions. We investigated both spacelike and timelike regions of the meson EM form factor using an exactly solvable model of $(3+1)$ dimensional scalar field theory interacting with gauge fields (see Eq. (1)).

We calculated the form factor in spacelike region using the Drell-Yan-West $\left(q^{+}=0\right)$ frame 
and showed that its analytic continuation to the timelike region reproduces exactly the direct result of timelike form factor obtained in the longitudinal momentum $\left(q^{+} \neq 0\right.$ and $\left.q_{\perp}=0\right)$ frame. The analytic continuation of the result in the $q^{+}=0$ frame to the timelike region automatically generates the effect of the nonvalence contributions to the timelike form factor. Another interesting result in our model calculations is that the peaks analogous to the VMD were generated and the position of peaks were indeed quite consistent with the VMD. Even though phenomenologically more elaborate analyses including the final state interaction may be necessary to reproduce the more realistic feature of VMD, our results seem pretty encouraging for further investigations. Using the dispersion relations, we have also confirmed that our numerical results of imaginary parts start at $q_{\min }^{2}=4 m_{q(\bar{Q})}$ and the normal thresholds appear for $\pi$ and $K\left(M^{2}<m_{q}^{2}+m_{\bar{Q}}^{2}\right)$ systems while the anomalous threshold exists for $D\left(M^{2}>m_{q}^{2}+m_{\bar{Q}}^{2}\right)$ system. However, we note that our form factor calculations are effectively various ways of evaluating the Feynman perturbation theory triangle diagrams and thus the analytic properties presented in sections II, III, and IV are restricted to one-loop diagrams.

Even though the model (Eq. (四)) may not be as realistic as one may want, we think that it plays the role of some guidance for the more realistic phenomenological models. For an application of our analytic continuation method to the more realistic model, we have considered an immediate extension of bosonic contact interaction to the fermionic covariant model with the monopole smearing vertices used by de Melo et al. [2,9] and investigated singular free processes in timelike region such as $0^{-}\left(=J^{P}\right) \rightarrow 0^{-}$and $0^{-} \rightarrow 1^{-}$semileptonic decays. In this application, we found that the covariant solution of the weak form factors $f_{+}$for $0^{-} \rightarrow 0^{-}$and $V$ and $A_{2}$ for $0^{-} \rightarrow 1^{-}$processes are exactly equal to our analytic solutions obtained from the current $j^{+}$in $q^{+}=0$ frame where only valence diagram is needed. On the other hand, the form factors $f_{-}$for $0^{-} \rightarrow 0^{-}$and $A_{0}$ and $A_{1}$ for $0^{-} \rightarrow 1^{-}$ processes turn out to be affected by the zero-mode contributions and one has to include the zero-mode effects to make our analytic solutions from $q^{+}=0$ frame equal to the covariant solutions (see also [10]). This covariant model calculation further verifies the validity of 
our analytic continuation method. We also applied our analytic continuation method to the same semileptonic decay processes of various pseudoscalar and vector mesons using our LFQM [7,21,22] and found a good agreement with the available experimental data as well as the lattice calculations.

However, an additional step of identifying the zero-mode contributions is necessary in handling the zero-mode issue associated with the currents $j_{\perp}$ for $0^{-} \rightarrow 0^{-}$and $j^{+}$coupled with longitudinal decay modes for $0^{-} \rightarrow 1^{-}$exclusive semileptonic decay processes. Also, the singular structures around the pole-regions in the timelike elastic form factors needs to be further investigated in the more phenomenological model. Nevertheless, it is encouraging that in this first attempt of calculating the timelike elastic meson form factor, the analytic continuation method seems to work as nicely as one may have anticipated.

We would like to thank G.-H.Kim for assisting the numerical analysis of dispersion relations. This work was supported by the U.S. DOE under contracts DE-FG02-96ER40947. The North Carolina Supercomputing Center and the National Energy Research Scientific Computer Center are also acknowledged for the grant of supercomputer time.

[1] G. P. Lepage and S. J. Brodsky, Phys. Rev. D 22 (1980) 2157.

[2] J. P. B. C. de Melo, J. H. O. Sales, T. Frederico, and P. U. Sauer, Nucl. Phys. A 631 (1998) $574 \mathrm{c}$.

[3] H.-M. Choi and C.-R. Ji, Phys. Rev. D 59 (1999) 034001.

[4] P. L. Chung, F. Coester, and W. N. Polyzou, Phys. Lett. B 205 (1988) 545.

[5] W. Jaus, Phys. Rev. D 44 (1991) 2851.

[6] F. Cardarelli et al., Phys. Lett. B 332 (1994) 1; Phys. Rev. D 53 (1996) 6682.

[7] H.-M. Choi and C.-R. Ji, Phys. Rev. D 59 (1999) 074015; ibid. 56 (1997) 6010. 
[8] B. D. Keister, Phys. Rev. D 49 (1994) 1500.

[9] J. P. B. C. de Melo, T. Frederico, H. W. L. Naus, and P. U. Sauer, Nucl. Phys. A 660 (1999) 219.

[10] W. Jaus, Phys. Rev. D 60 (1999) 054026.

[11] S. J. Brodsky and D. S. Hwang, Nucl. Phys. B 543 (1998) 239.

[12] M. Sawicki and L. Mankiewicz, Phys. Rev. D 37 (1988) 421; L. Mankiewicz and M. Sawicki, ibid. 40 (1989) 3415.

[13] S. Glazek and M. Sawicki, Phys. Rev. D 41 (1990) 2563.

[14] M. Sawicki, Phys. Rev. D 44 (1991) 433; ibid. 46 (1992) 474.

[15] H.-M. Choi and C.-R. Ji, Phys. Rev. D 58 (1998) 071901.

[16] N.C.J. Schoonderwoerd and B.L.G. Bakker, Phys. Rev. D 57 (1998) 4965; ibid. 58 (1998) 025013.

[17] J. D. Bjorken and S. D. Drell, Relativistic Quantum Fields (McGraw-Hill, New York, 1965), pp. 209-282.

[18] S. Gasiorowicz, Elementary Particle Physics (Wiley, New York, 1966), pp. 348-362.

[19] S. Mandelstam, Phys. Rev. Lett. 4 (1960) 84.

[20] M. Gell-Mann, M. L. Goldberger, and W. Thirring, Phys. Rev. 95 (1954) 1612; M. Goldberger, Phys. Rev. 99 (1955) 979.

[21] H.-M. Choi and C.-R. Ji, Phys. Lett. B 460 (1999) 461.

[22] H.-M. Choi, Ph.D. Thesis. hep-ph/9911271. 


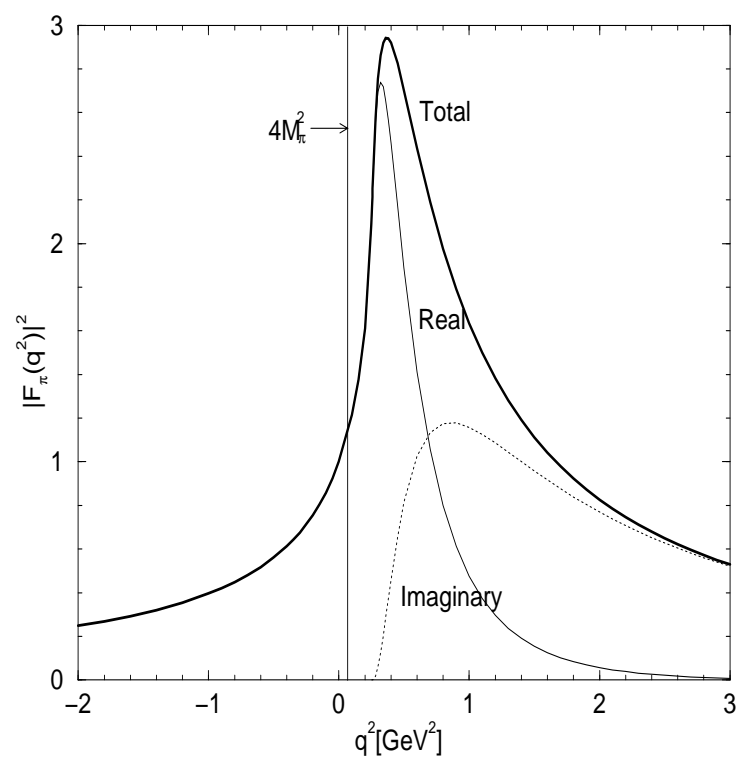

FIG. 4.a. The electromagnetic form fator of the pion in $(3+1)$ dimensional scalar field theory for $-2 \leq q^{2} \leq 3 \mathrm{GeV}^{2}$. The total, real, and imaginary parts of $\left|F_{\pi}\left(q^{2}\right)\right|^{2}$ are represented by thick solid, solid, and dotted lines, respectively.

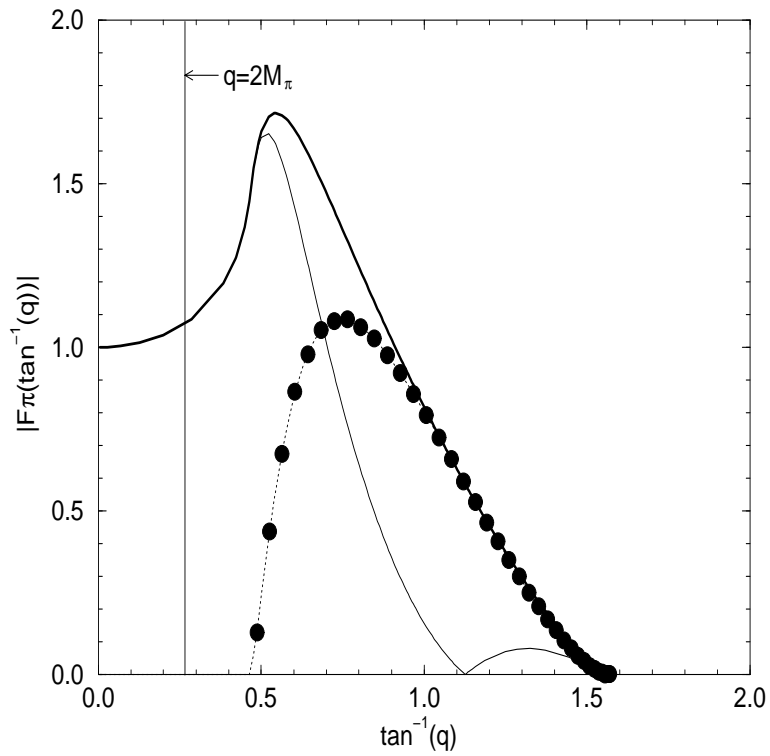

FIG. 4.b. The electromagnetic form fator of the pion in $(3+1)$ dimensional scalar field theory for the entire timelike region compared to the dispersion relations (data of black dots) given by Eq. (25). The same line code as in Fig. 4(a) is used. 


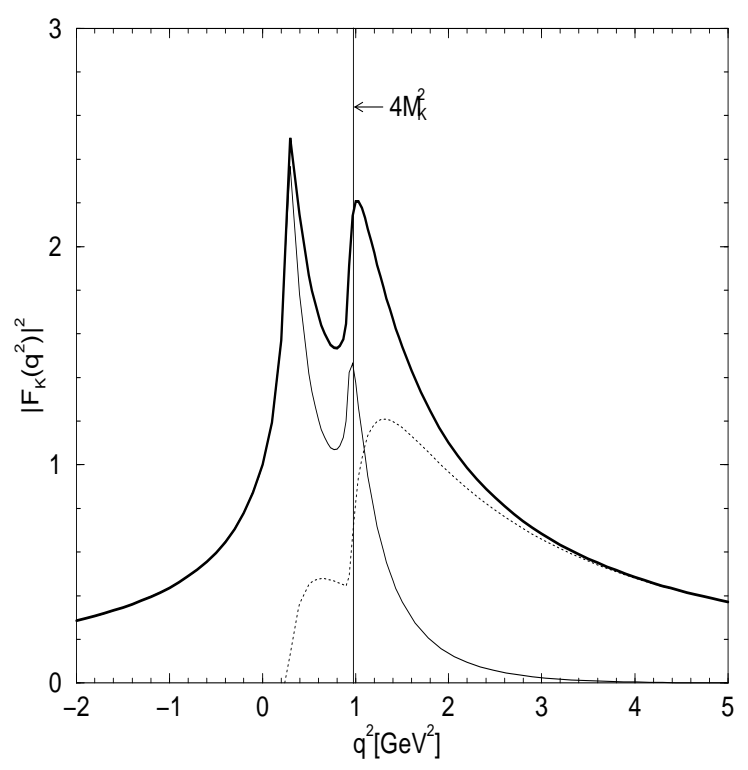

FIG. 5.a. The electromagnetic form fator of the kaon in $(3+1)$ dimensional scalar field theory for $-2 \leq q^{2} \leq 5 \mathrm{GeV}^{2}$. The same line code as in Fig. 4(a) is used.

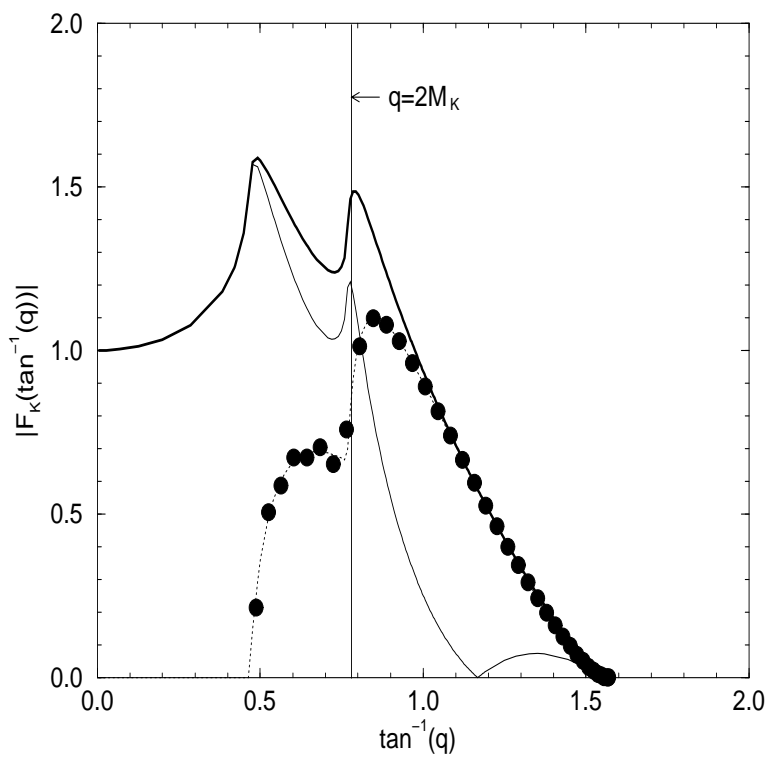

FIG. 5.b. The electromagnetic form fator of the kaon in $(3+1)$ dimensional scalar field theory for the entire timelike region compared to the dispersion relations (data of black dots) given by Eq. (25). The same line code as in Fig. 4(a) is used. 


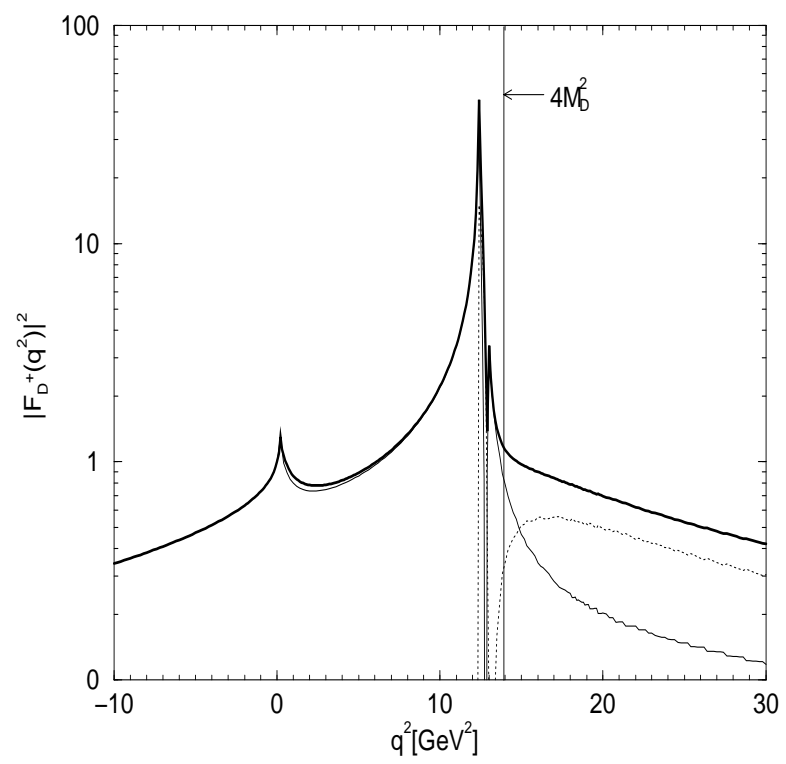

FIG. 6. The electromagnetic form fator of the $D$ meson in $(3+1)$ dimensional scalar field theory for $-10 \leq q^{2} \leq 30 \mathrm{GeV}^{2}$. The same line code as in Fig. 4(a) is used. 